\section{Adaptación y evaluación inicial del Charing Cross Venous Ulcer Questionnaire en pacientes con úlceras venosas crónicas en Uruguay}

\author{
GABRIELA TAFERNABERRY ${ }^{1}$, GABRIELA OTERO ${ }^{1,2}$, \\ CAROLINE AGORIO ${ }^{1,2}$, JUAN J. DAPUETO ${ }^{1,3}$
}

\section{Evaluation of the Charing Cross Venous Ulcer Questionnaire in patients with chronic venous ulcers in Uruguay}

Background: Chronic venous ulcers (CVU) represent a frequent condition, with difficult therapeutic approaches, that impact on patients' quality of life, and generate an economic burden to patients and health systems. Aim: To perform the cultural adaptation and initial evaluation of the Charing Cross Venous Ulcer Questionnaire (CCVUQ) for Uruguay, and to study the health-related quality of life (HRQL) of patients with CVU. Material and Methods: The translated and culturally adapted version of the CCVUQ was applied to a convenience sample of 50 patients. In addition, the PROMIS Global Health Survey was included in the assessment. Results: Both questionnaires showed good internal consistency (Cronbach alfa $>0.70$ ). A statistically significant association was observed between the CCVUQ total scores, its subscales and both dimensions of the PROMIS: Global Physical (GPH) and Global Mental Health (GMH) (rho $\geq 0.40$ ). The CCVUQ mean score was $54.9 \pm 42$ points while GPH and GMH mean scores were $37.9 \pm 29$ points, and $43.1 \pm 35.1$ points respectively. Simple linear regression showed that patients with higher income reported better emotional well-being, while in younger patients, ulcers had a higher impact on Emotional Status and Cosmetics. Conclusions: The translated and adapted version of the CCVUQ was easy to comprehend and apply, showing good psychometric properties. When used in association with the PROMIS Global Health Measure it provides complementary information. HRQL was severely affected in the study sample.

(Rev Med Chile 2016; 144: 55-65)

Key words: Leg ulcer; Quality of life; Questionnaires; Validation studies.

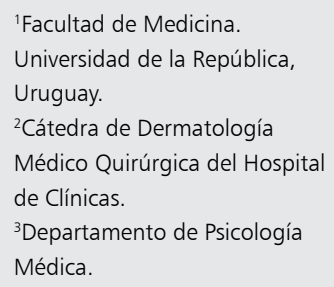

Los autores declaran no tener ningún subsidio ni apoyo financiero, así como tampoco existió influencia alguna en el diseño del estudio por parte de terceros.

Recibido el 20 de marzo de 2015 aceptado el 15 de octubre de 2015.

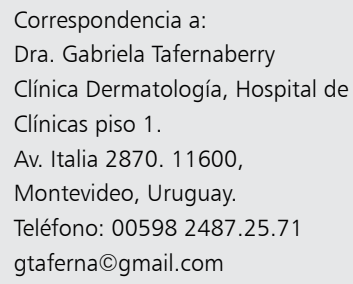

L as úlceras venosas crónicas de miembros inferiores (UVC) son una patología frecuente, con una incidencia de 3 a 5 nuevos casos por mil personas por año. Presentan una relación mujer/hombre de $3: 1^{1}$, con una prevalencia de 0,1 a $0,3 \%$ en la población europea, aumentando a $1 \%$ en mayores de 65 años ${ }^{2-8}$.

Las UVC generan un complejo conjunto de síntomas como dolor, limitación de la movilidad, molestias por la herida (exudado, mal olor, infecciones) que impactan severamente en la calidad de vida9. Pueden determinar cambios en la autopercepción con sentimientos de vergüenza, pérdida de feminidad, trastornos del estado de ánimo y del sueño, limitación de la vida social, de las actividades recreativas y laborales, imponiendo 
limitaciones en el vestir, con esfuerzos para ocultar la úlcera y las curaciones voluminosas ${ }^{10,14}$. Otros factores como la edad, el sexo y el estatus socioeconómico del paciente pueden atenuar o por el contrario agravar, el impacto de estas sobre la calidad de vida ${ }^{9,10,12,15}$.

El concepto de la calidad de vida relacionado con la salud (CVRS) ha despertado gran interés en diversas áreas de la medicina en los últimos 20 años $^{16}$. Este enfoque se basa no solamente en tratar la enfermedad sino en promover el bienestar del paciente $e^{13,17,18}$. Se la ha definido como "la medida en la cual el bienestar físico, emocional o social habitual o esperado, se afecta como consecuencia de una enfermedad médica o por su tratamiento"19. Se la considera un concepto subjetivo y multidimensional, que está determinado por los efectos de la enfermedad y el tratamiento instituido en las múltiples dimensiones: física, emocional, social, familiar, funcional y espiritual ${ }^{17,18,20}$. Al destacarse la subjetividad, las evaluaciones que hace el sujeto pueden no corresponderse con las realizadas por los clínicos, basadas exclusivamente en criterios biomédicos $^{18,21,24}$. Las mediciones de CVRS constituyen una forma de resultados evaluados por los pacientes (patient-reported outcomes o PROS, en inglés), entendidos como los reportes que hace el sujeto sobre aspectos relacionados con su salud o con los tratamientos sin la intervención externa del médico o cualquier otra persona. La Administración de Alimentos y Drogas de Estados Unidos (FDA) ha establecido guías para los estudios enfocados en $P R O S^{25}$.

A lo largo de los años, se han desarrollado diversos instrumentos para evaluar la CVRS tanto genéricos como específicos para $\mathrm{UVC}^{26,30}$. Un listado de ellos se presenta en la Tabla 1.

Dada la diversidad en cuanto a la percepción de salud y enfermedad entre las diferentes poblaciones se hace imprescindible que los instrumentos sean validados transculturalmente, asegurando que sus mediciones sean equivalentes a las obtenidas en las poblaciones para los cuales fueron diseñados originalmente ${ }^{38,46-50}$.

En nuestro país, no existen estudios sobre CVRS para UVC, ni instrumentos confiables y válidos para su aplicación. En esta comunicación se presenta el proceso de adaptación transcultural y la evaluación de las propiedades psicométricas y de uso clínico del cuestionario de calidad de vida para úlceras venosas Charing
Cross Venous Ulcer Questionnaire (CCVUQ), versión para Uruguay.

\section{Materiales y Métodos}

El estudio presentó dos fases, una primera de traducción y adaptación transcultural del CCVUQ y una segunda fase para evaluación de las propiedades psicométricas y su aplicabilidad clínica.

\section{Instrumentos}

Previa a la elección del instrumento se tuvo en cuenta la disponibilidad de cuestionarios. Se optó por CCVUQ ya que estudios anteriores en población de habla hispana reportaron buenas propiedades psicométricas, fácil aplicación, con un tiempo de administración de $15 \mathrm{~min}$. Consta de veintiuna preguntas agrupadas en cinco dimensiones: Interacción social, Actividades domésticas, Función o Actividad social, Aspectos estéticos o Cosmesis y Estado emocional. Proporciona un puntaje total que es la suma de las subescalas. Utiliza una escala de valoración tipo Likert con 5 y 6 niveles. Los puntajes son transformados de $0 \mathrm{y}$ 100 para su mejor comparación; las puntuaciones mayores indican peor calidad de vida. Es de uso libre bajo autorización y se descarga de manual de aplicación y puntación desde MAPI Research Institute (Instituto de Investigación MAPI) ${ }^{51}$. El punto de partida para la adaptación al español del Río de la Plata fue la versión original en inglés ${ }^{43}$.

Se aplicó además un instrumento genérico de bienestar físico y emocional, el Patient Reported Outcomes Measurement Information System (PROMIS) Global Health Measure (PGHM) (Medida Global de Salud del Sistema de Información de Medida de Resultados Reportados por el Paciente) que consta de 10 ítems agrupados en una escala de Salud Física Global (SFG) y una escala de Salud Mental Global (SMG), más dos ítems: bienestar social y calidad de vida global ${ }^{52}$. Es de uso libre previa autorización. De la misma forma que CCVUQ este cuestionario debe ser respondido en su totalidad para poder ser puntuado.

Los puntajes del PROMIS son transformados a puntajes T, para poder ser comparados con la norma de la población de EE. UU. (50 puntos corresponden a la media de la población y 10 puntos a un desvío estándar). Mayores puntuaciones indican mejor CVRS ${ }^{40,47,53}$. 


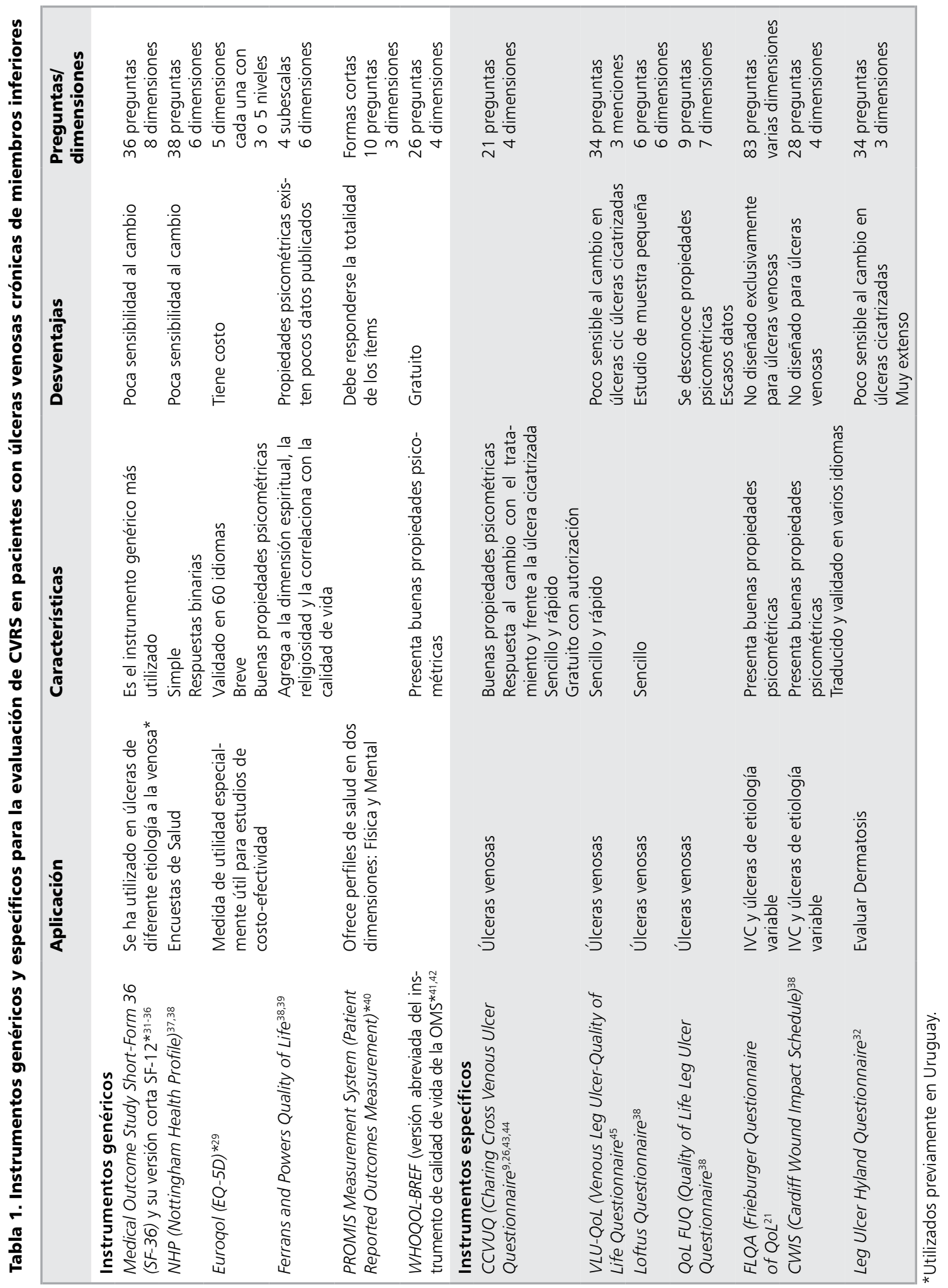




\section{Fase de traducción y adaptación transcultural}

Se realizó siguiendo pautas internacionales ${ }^{54,58}$. Se realizaron dos traducciones independientes del idioma original (fuente) al idioma local (idioma diana), por traductores de idioma nativo español. Ambas versiones, se conciliaron entre sí, obteniendo una primera versión en el idioma local. A continuación, se realizó una retro-traducción al idioma fuente, por traductor de idioma nativo inglés. De esta primera retro-traducción se obtuvieron dos nuevas traducciones independientes al idioma local que se conciliaron entre sí, dando lugar a la segunda versión definitiva en español local. Por último, se realizó un testado piloto en diez pacientes en base a una entrevista estructurada cognitiva (cognitive debriefing) para evaluar la comprensión y facilidad de aplicación (Figura 1).

\section{Pacientes}

Se seleccionó una muestra de conveniencia constituida por todos los pacientes portadores de UVC, mayores de 18 años, que consultaron entre marzo y octubre de 2012 en la Policlínica de Úlceras de la Cátedra de Dermatología del Hospital de Clínicas, tanto por primera vez como para control clínico. Fueron excluidos los pacien-

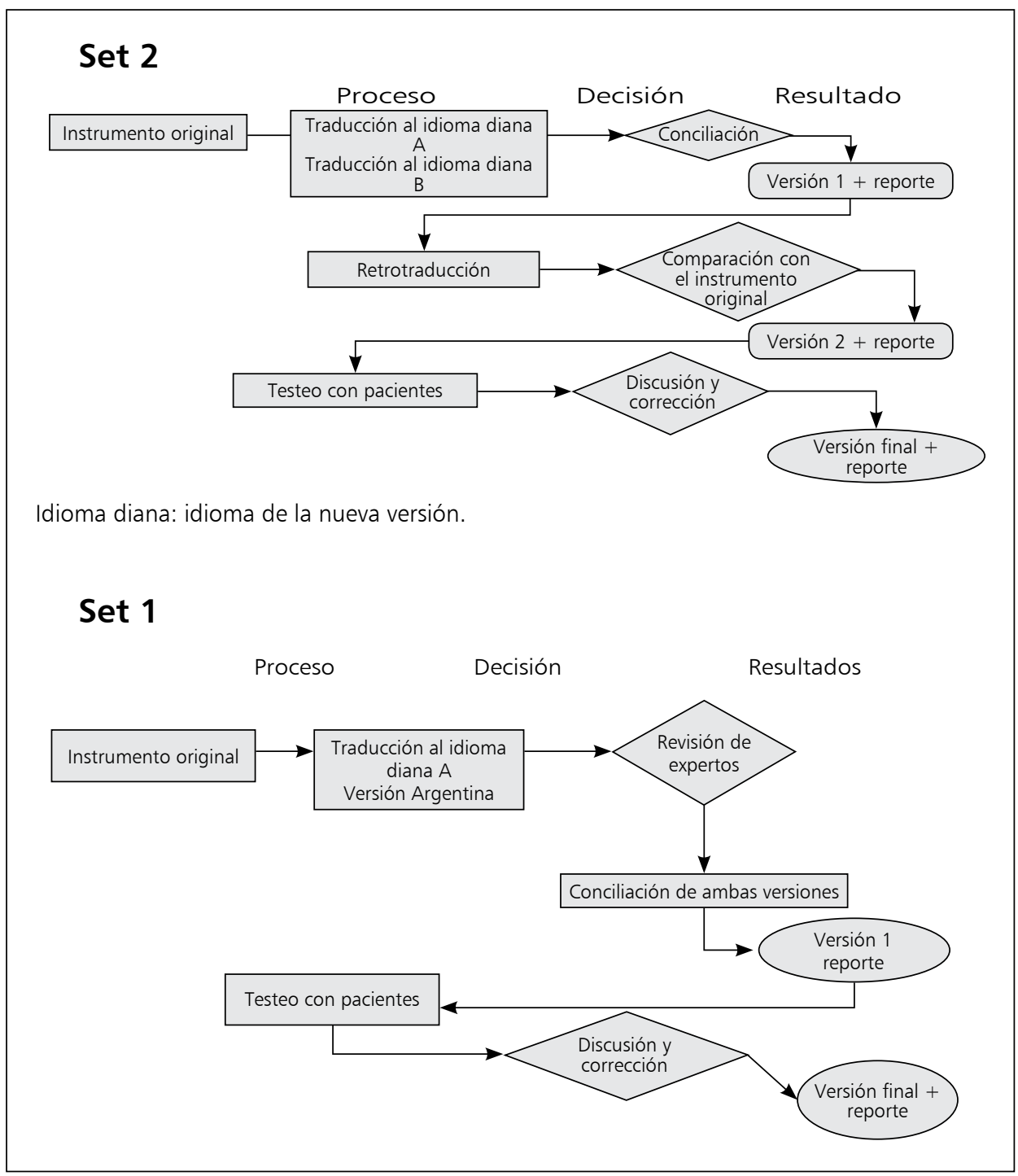

Figura 1. Esquema de validación del CCVUQ. 
tes portadores de úlceras de etiología no venosa, con diabetes mellitus o enfermedad arterial, pacientes terminales, con deterioro cognitivo o con dificultad severa en la comunicación o lectura. Se registraron datos epidemiológicos, biomédicos, sociodemográficos y comorbilidades, proporcionados por los pacientes o extraídos de las historias clínicas. Se obtuvo aprobación por el Comité de Ética de la Investigación del Hospital de Clínicas, solicitándose consentimiento informado previo a la participación.

\section{Análisis estadístico}

Se utilizaron procedimientos de la estadística descriptiva e inferencial.

Se estudió la confiabilidad mediante el coeficiente alfa de Cronbach, para cada subescala y para la escala global del CCVUQ. Se consideró aceptable un valor de 0,70 o mayor.

Se estudió la validez de constructo mediante el cálculo de las correlaciones no paramétricas de Spearman entre los puntajes de los instrumentos. Se espera que instrumentos que miden constructos similares tengan alta correlación entre sí (validez convergente). Se evaluó además la capacidad de los cuestionarios de discriminar entre grupos de pacientes que a priori se espera difieran en su CVRS (diferencia entre grupos conocidos). Para ello se consideró una serie de variables biomédicas y psicosociales que teóricamente pueden influir en la CVRS, las cuales se presentan en las Tablas $2 \mathrm{y}$ 3. Se realizó el cálculo de coeficientes de regresión lineal simple de los puntajes de los instrumentos (totales y subescalas) para cada una de las variables biomédicas y sociodemográficas.

\section{Resultados}

Fueron abordados 52 pacientes, dos pacientes se negaron a participar del estudio (tasa de respuesta 96,1\%). En 49 de los 50 casos, los pacientes respondieron en forma completa todas las escalas y subescalas. De incluidos, 24 hombres $(48 \%)$ y 26 mujeres (52\%) con una media de edad de 63,4 años (rango: $34-84$ años). El 64\% concurría a la policlínica desde hacía más de un año y el $18 \%$ desde hacía 5 años o más. El 68\% (n=34) de los pacientes había iniciado su primera úlcera hacía 10 años o más, mientras que la úlcera actual presentaba un tiempo de evolución mayor a un año en el $64 \%(n=32)$ de los pacientes. Se trató de una muestra con bajo nivel de instrucción (sólo 6\% había completado el segundo ciclo de secundaria o más), con bajo nivel económico ( $80 \%$ de los pacientes refería que sus ingresos no le alcanzaban para cubrir sus necesidades o que sufrían penurias) y con múltiples comorbilidades: obesidad (48\%), ser fumador o ex fumador (44\%), hipertensión arterial (HTA) (56\%), cardiopatía (32\%) o fracturas de miembros inferiores (20\%). Reportaron síntomas de insuficiencia venosa crónica (IVC) $80 \%$ de los pacientes tales como edema gravitacional, piernas cansadas, visualización de trayectos varicosos, entre otros. Las frecuencias, medias y desvíos estándar de las variables biomédicas y sociodemográficas se muestran en las Tablas 2 y 3.

Todos los instrumentos fueron aplicados en una única sesión. Del total de pacientes, 37 (74\%) completó el formulario en forma autoadministrada mientras que 13 (26\%) prefirió que el mismo fuera leído por el entrevistador debido a dificultad para la lectura o problemas visuales.

La Tabla 4 muestra los coeficientes alfa de Cronbach y estadísticos descriptivos de los puntajes totales y de subescala del CCVUQ y de PGHM.

Tabla 2. Variables biomédicas

\begin{tabular}{|c|c|c|}
\hline & $\mathbf{n}$ & $\%$ \\
\hline $\begin{array}{l}\text { Tiempo de } 1^{\circ} \text { episodio* } \\
\quad<\text { de } 10 \text { años } \\
\geq 10 \text { años }\end{array}$ & $\begin{array}{l}34 \\
16\end{array}$ & $\begin{array}{l}68 \\
32\end{array}$ \\
\hline $\begin{array}{l}\text { Tiempo evolución úlcera actu } \\
\text { Actual }<\text { de } 12 \text { meses } \\
\geq \text { de } 12 \text { meses }\end{array}$ & $\begin{array}{l}18 \\
32\end{array}$ & $\begin{array}{l}36 \\
64\end{array}$ \\
\hline $\begin{array}{l}\text { n de úlceras } \\
1 \text { úlcera } \\
>1 \text { úlcera }\end{array}$ & $\begin{array}{l}35 \\
15\end{array}$ & $\begin{array}{l}70 \\
30\end{array}$ \\
\hline Tamaño medio úlcera & $49,92 \mathrm{~cm}^{2}$ & (SD 56,6) \\
\hline $\begin{array}{l}\text { Concurre a control } \\
\quad \leq 1 \text { año } \\
>1 \text { año }\end{array}$ & $\begin{array}{l}27 \\
23\end{array}$ & $\begin{array}{l}54 \\
46\end{array}$ \\
\hline $\begin{array}{l}\text { Presenta complicaciones } \\
\text { No presenta complicaciones }\end{array}$ & $\begin{array}{l}30 \\
20\end{array}$ & $\begin{array}{l}60 \\
40\end{array}$ \\
\hline $\begin{array}{l}\text { Usa vendaje elástico } \\
\text { No usa vendaje elástico }\end{array}$ & $\begin{array}{l}28 \\
22\end{array}$ & $\begin{array}{l}56 \\
44\end{array}$ \\
\hline $\begin{array}{l}\text { Autoválido } \\
\text { No autoválido }\end{array}$ & $\begin{array}{r}47 \\
3\end{array}$ & $\begin{array}{r}94 \\
6\end{array}$ \\
\hline
\end{tabular}

*Tiempo del episodio inicial de úlcera venosa crónica. 
Tabla 3. Variables epidemiológicas

\begin{tabular}{|c|c|c|}
\hline & $n^{*}$ & $\%$ \\
\hline \multicolumn{3}{|l|}{ Edad } \\
\hline$<50$ & 6 & 12 \\
\hline $50-64$ & 20 & 40 \\
\hline$\geq 65$ & 24 & 48 \\
\hline \multicolumn{3}{|l|}{ Sexo } \\
\hline Mujer/Hombre & $26 / 24$ & $52 / 48$ \\
\hline \multicolumn{3}{|l|}{ Procedencia } \\
\hline Montevideo/Interior & $34 / 16$ & $68 / 32$ \\
\hline \multicolumn{3}{|l|}{ Estado civil } \\
\hline Casado & 17 & 34 \\
\hline Unión libre & 7 & 14 \\
\hline Separado & 6 & 12 \\
\hline Viudo & 6 & 12 \\
\hline Soltero & 8 & 16 \\
\hline Divorciado & 6 & 12 \\
\hline \multicolumn{3}{|l|}{ Nivel de instrucción } \\
\hline$<3$ años primaria & 8 & 16,0 \\
\hline De 3 a 6 años de primaria & 16 & 32,0 \\
\hline Primaria completa & 8 & 16,0 \\
\hline Secundario primer ciclo & 12 & 24,0 \\
\hline Secundario segundo ciclo & 4 & 8,0 \\
\hline Terciario & 2 & 4 \\
\hline \multicolumn{3}{|l|}{ Composición grupo familiar } \\
\hline Vive solo & 14 & 20 \\
\hline Vive con familiar & 31 & 62,0 \\
\hline Vive con otro & 4 & 8,0 \\
\hline Vive en residencial & 1 & 2,0 \\
\hline \multicolumn{3}{|l|}{ Ingreso familiar mensual } \\
\hline$\leq$ salario mínimo** & 33 & 66 \\
\hline > salario mínimo** & 17 & 34 \\
\hline \multicolumn{3}{|l|}{ Mínimo 0} \\
\hline \multicolumn{3}{|l|}{ Máximo $\$ 40.000$} \\
\hline Promedio $\$ 7,586$. SD $\$ 6,854$ & & \\
\hline
\end{tabular}

\begin{tabular}{|c|c|c|}
\hline & $\mathbf{n}^{*}$ & $\%$ \\
\hline \multicolumn{3}{|l|}{ Ocupación } \\
\hline $\begin{array}{l}\text { Trabajo remunerado, jornada } \\
\text { completa }\end{array}$ & 2 & 4,0 \\
\hline $\begin{array}{l}\text { Trabajo remunerado, jornada } \\
\text { incompleta }\end{array}$ & 11 & 22,0 \\
\hline Jubilado, pensionista & 24 & 48,0 \\
\hline Desocupado & 11 & 22,0 \\
\hline Ama de casa & 1 & 2,0 \\
\hline \multicolumn{3}{|l|}{ Religión } \\
\hline No profesa & 17 & 34 \\
\hline Sí profesa & 32 & 64 \\
\hline \multicolumn{3}{|l|}{ Tipo de religión } \\
\hline Católica & 16 & 50 \\
\hline Protestante & 1 & 3 \\
\hline Evangélica & 5 & 16 \\
\hline Judía & 1 & 3 \\
\hline Afrouruguaya & 1 & 3 \\
\hline No Especifica & 8 & 25 \\
\hline \multicolumn{3}{|l|}{ Fuma } \\
\hline No fuma & 28 & 56 \\
\hline Ex fumador & 17 & 34 \\
\hline Fuma actualmente & 5 & 10 \\
\hline \multicolumn{3}{|l|}{ Consumo de alcohol } \\
\hline $\begin{array}{l}\text { No consume o consumo de bajo } \\
\text { riesgo }\end{array}$ & 45 & 90 \\
\hline Ex bebedor de riesgo & 4 & 8 \\
\hline Consumo de alcohol de riesgo actual & 1 & 2 \\
\hline Obesidad & 24 & 48 \\
\hline Cardiopatía & 16 & 32 \\
\hline Síntomas de IVC & 40 & 80 \\
\hline TVP & 14 & 28 \\
\hline HTA & 28 & 56 \\
\hline
\end{tabular}

*N Total 50 pacientes. **Salario mínimo SM-Instituto Nacional de Estadística correspondiente a junio de 2013 pesos uruguayos $\$ 7,000$. SD. Desvío standard. IVC: Insuficiencia venosa crónica. TVP: trombosis venosa profunda. HTA: hipertensión arterial.

Tabla 4. Alfa de Cronbach, medias y Desvío Estándar, Mediana de CCVUQ y PROMIS Global Health

\begin{tabular}{|lccccccccc|}
\hline & n & Alfa Cronbach & Media & P25 & P75 & Mediana & Mínimo & Máximo & DE \\
\hline PROMIS SFG & 50 & 0,73 & 37,9 & 32,4 & 42,9 & 37,4 & 16,20 & 57,70 & 28,9 \\
PROMIS SMG & 49 & 0,75 & 43,1 & 37,5 & 48,3 & 43,5 & 21,20 & 59,00 & 35,1 \\
AVSCORE & 50 & 0,83 & 54,9 & 46,0 & 63,0 & 56,0 & 21,00 & 88,00 & 42,0 \\
INT-SOC & 49 & 0,61 & 45,1 & 33,0 & 55,0 & 45,0 & 17,00 & 86,00 & 28,6 \\
ACT-DOM & 50 & 0,76 & 43,1 & 25,5 & 54,5 & 41,1 & 5,00 & 84,00 & 21,7 \\
COSMES & 50 & 0,70 & 62,4 & 48,0 & 73,2 & 64,0 & 24,00 & 95,00 & 45,4 \\
\hline EST-EMO & 50 & 0,71 & 70,6 & 54,8 & 84,5 & 75,5 & 21,00 & 100,00 & 51,7 \\
\hline
\end{tabular}

SFG: PROMIS Salud Física Global; SMG: PROMIS Salud Mental Global; AVSCORE: Puntaje total del CCVUQ; INT-SOC: Interacciones sociales; ACT-DOM: Actividades domésticas; COSMES: Cosmesis, EST-EMO: Estado Emocional. 
Tabla 5. Matriz de coeficientes de correlación de spearman

\begin{tabular}{|c|c|c|c|c|c|c|c|}
\hline & AVUSCORE & SOCINT & DOMACT & COSMES & EMSTAT & $\begin{array}{l}\text { PROMIS } \\
\text { GLOBAL } \\
\text { PHYSICAL } \\
\text { SCALE }\end{array}$ & $\begin{array}{c}\text { PROMIS } \\
\text { GLOBAL } \\
\text { EMOTIONAL } \\
\text { SCALE }\end{array}$ \\
\hline AVUSCORE & 1 & $0,69 * * *$ & $0,61 * * *$ & $0,77 * * *$ & $0,74^{* * *}$ & $0,66^{* * *}$ & $0,47^{* * *}$ \\
\hline INT-SOC & & 1 & 0,29 * & $0,29 *$ & $0,30 *$ & $-0,40$ ** & $-0,232$ \\
\hline ACT-DOM & & & 1 & 0,257 & 0,144 & $-0,182$ & $-0,229$ \\
\hline COSMES & & & & 1 & 0,746 & $-0,617$ & $-0,428$ \\
\hline EST-EMO & & & & & 1 & $-0,598$ & $-0,42$ \\
\hline SFG & & & & & 1 & 1 & 0,472 \\
\hline SMG & & & & & & & 1 \\
\hline
\end{tabular}

AVSCORE: Puntaje total del CCVUQ; INT-SOC: Interacciones sociales; ACT-DOM: Actividades domésticas; COSMES: Cosmesis; EST-EMO: Estado Emocional; SFG: PROMIS Salud Física Global; SMG: PROMIS Salud Mental Global. ${ }^{* * p}<0,001$; ${ }^{* *} p<0,01 ;{ }^{*} p<0,05$

Excepto en la subescala de Interacciones sociales, los coeficientes de confiabilidad fueron iguales $o$ mayores de 0,70 .

La media y mediana de los puntajes de la escala de SFG del PGHM se encuentra 1,2 desvíos estándar (DE) por debajo de la norma de la población de Estados Unidos de Norteamérica (rango: 16,2 a 57,7 puntos), lo que indica una afectación importante del bienestar físico de estas personas. La afectación de la esfera emocional es menos severa pero igualmente importante con valores de media y mediana cercanos a 0,6 DE por debajo de la media poblacional de referencia.

La Tabla 5 muestra la matriz de coeficientes de correlación de Spearman entre los puntajes de los instrumentos. Se observa que existe una fuerte asociación estadísticamente significativa entre los puntajes totales del CCVUQ con sus subescalas y con las dos dimensiones del PGHM (coeficientes rho $\geq 0,40$ ), así como entre las dimensiones Cosmesis, Estado Emocional e Interacciones Sociales y ambas dimensiones del PROMIS (los coeficientes negativos se deben a las diferentes formas de puntuación de los cuestionarios). Estos resultados evidencian una buena validez convergente.

En cuanto a la diferencia entre grupos conocidos, la regresión lineal simple mostró que los pacientes con mayores ingresos del núcleo familiar presentaban mejor bienestar emocional evaluado por la escala SMG del PROMIS (coeficiente de regresión $\mathrm{B}: 4,82$, coeficiente $\beta$ estandarizado $\mathrm{p}=0,046$, Error típico: 2,35).
En cuanto a la percepción del Estado Emocional y la Cosmesis evaluado por el CCVUQ, se evidenció que los pacientes más jóvenes presentaban mayor afectación emocional (Subescala Estado emocional (coeficiente de regresión B: -7,8, Error típico: 3,78, coeficiente $\beta$ estandarizado: -,28, $\mathrm{p}=0,044)$ y mayor impacto estético (Subescala Cosmesis coeficiente de regresión B -7,224, coeficiente $\beta$ estandarizado: -,29, Error típico: $3,39, \mathrm{p}=0,038)$. En el resto de los casos los coeficientes de regresión no alcanzaron el nivel de significación estadística.

\section{Discusión}

La muestra evaluada presentó UVC recurrentes y de larga evolución, representando altos costos sanitarios para las instituciones ${ }^{3,5}$.

El proceso de adaptación transcultural al español del Río de la Plata del CCVUQ culminó con una versión fácil de comprender, aplicar y procesar. Respecto a la validez de contenido, el CCVUQ cubre un amplio rango de dimensiones relevantes para la CVRS que abarca las áreas social, emocional, de las actividades diarias e imagen corporal. Se ha reportado el impacto de las UVC sobre la sexualidad ${ }^{59}$, dimensión que no está incluida en el cuestionario.

La distribución de puntajes del CCVUQ total y por dimensiones abarcó un amplio rango de puntuaciones, sin que se evidenciara efecto piso 
o techo. Las dimensiones más afectadas fueron Estado Emocional y Cosmesis.

Las propiedades psicométricas mostraron una adecuada confiabilidad con coeficientes alfa de Cronbach en rangos de aceptable a bueno tanto para el cuestionario total como para sus subescalas. Presenta además adecuada validez convergente con altas correlaciones con el PGHM en sus dos dimensiones.

Sin embargo, en la muestra estudiada, no se pudo demostrar adecuada validez en cuanto a la discriminación entre grupos conocidos. Sólo las escalas de Estado Emocional y Cosmesis pudieron discriminar entre los pacientes de acuerdo a la edad, observándose que los pacientes más jóvenes reportaron peor calidad de vida. La relación entre la edad y la CVRS ha sido estudiada en otras enfermedades crónicas con resultados variados ${ }^{60}$. En nuestro estudio este resultado podría atribuirse a que el impacto de la UVC (aislamiento social, pérdida de ingresos, baja autoestima, retraimiento) sería mayor en los pacientes más jóvenes.

El cuestionario PGHM confirmó su buena confiabilidad y validez reportados antes en muestras nacionales ${ }^{61}$.

En cuanto a aspectos clínicos, los pacientes evaluados tenían úlceras de larga evolución, gran tamaño, difícil cicatrización y recurrentes, lo que constituye factores de mal pronóstico ${ }^{65}$.

La edad y la distribución por sexo de la muestra estudiada se corresponde con lo reportado por otros autores $3,5,8,62-66$. En un estudio realizado en Brasil sobre UVC se encontraron resultados similares en media de edad e ingresos, comorbilidades, duración y porcentaje de recidivas ${ }^{67}$.

Los pacientes analizados presentan alta vulnerabilidad social, bajo nivel educativo y de ingresos. En este sentido, varios estudios mencionan la asociación entre mayor duración de la UVC y el medio social deficitario, constituyendo un factor predictivo negativo en su evolución ${ }^{68}$, siendo difícil determinar si la afectación socioeconómica es causa o consecuencia de la UVC ${ }^{69,70}$.

Como limitaciones del estudio, se trata de una muestra pequeña y homogénea en la mayoría de las variables estudiadas, lo que pudo haber quitado significación estadística al estudio de grupos conocidos. Por lo mismo, los resultados no pueden generalizarse a la totalidad de los pacientes portadores de UVC de otros centros de salud en los cuales la diversidad en los indicadores de educación, ingreso y sociales son diversos. Por este motivo se deben considerar estos aspectos en estudios futuros que permitan la correcta validación de las variables analizadas.

\section{Conclusión}

Las UVC son una patología frecuente con deterioro en la calidad de vida, pérdida de capacidad laboral e impacto sanitario importante. Evaluar la CVRS en estos pacientes contribuiría a optimizar los recursos destinados a los tratamientos especialmente en poblaciones vulnerables. La adaptación transcultural del CCVUQ culminó con la obtención de un instrumento de fácil aplicación y con características psicométricas que permitirán su inclusión en futuros estudios de CVRS en grupos más amplios de pacientes portadores de UVC.

\section{Referencias}

1. Otero G, Agorio C, Martínez M. Úlceras de miembros inferiores. Características clínico-epidemiológicas de los pacientes asistidos en la unidad de heridas crónicas del Hospital de Clínicas. Rev Méd Urug 2012; 28 (3): 182-9.

2. Buchanan $\mathrm{M}$. The impact of clinical factors and sociodemographic variables on health-related quality of life in venus leg ulceration. Disponible en: http://qspace. library.queensu.ca/bitstream/1974/5393/1/Buchanan_ Maureen_M_201001_MSc.pdf. [Consultado el 4 de agosto de 2015].

3. Rodríguez-Piñero M. Epidemiología, repercusión sociosanitaria y etiopatogenia de las úlceras vasculares. Angiología 2003; 55: 260-7.

4. Restrepo Medrano JC. Instrumentos de monitorización clínica y medida de la cicatrización en úlceras por presión (UPP) y úlceras de la extremidad inferior (UEI). Desarrollo y validación de un índice de medida. Tesis doctoral Universidad de Alicante; 2010. Disponible en: http://www.gneaupp.es/app/adm/publicaciones/archivos/40_pdf. [Consultado el 3 de marzo de 2013].

5. Lozano F, Jiménez Cossío JA, Ulloa J. La insuficiencia venosa crónica en España. Estudio epidemiológico RELIEF. Angiología 2001; 53: 5-16.

6. Álvarez Fernández LJ, Lozano F, Marinello Roura J, Masegosa, Medina JA. Encuesta epidemiológica sobre la insuficiencia venosa crónica en España. Angiología 2008; 60: 27-36.

7. Skovberg Roaldsen K, Biguet G, Elfving B. Physical 
activity in patients with venous leg ulcer-between engagement and avoidance. Clinic Rehabil 2011; 25: 275-86.

8. Flanagans M, Rotchell M, Fletcher J, Schofield J. Community nurses, home carers and patients perceptions of factors affecting venous leg ulcer recurrence and management of services. J Nurs Management 2001; 9: 153-9.

9. Martin-Paredero V. Epidemiología de la úlcera de etiología isquémica. Ann Pat Vasc 2009; 3: 55-75.

10. Finlayson K, Edwards H, Courtney M. The impact of psychosocial factors on adherence to compression therapy to prevent recurrence of venous leg ulcers. J Clin Nurs 2010; 19: 1289-97.

11. Heinen MM, Persoon A, van de Kerkhof P, Otero M, van Achterberg T. Ulcer-related problems and health care needs in patients with venous leg ulceration: a descritive, cross-sectional study International. Int J Nurs Stud 2007; 44 (8): 1296-303.

12. SoonK, Acton C. Pain-induced stress: a barrier to wound healing. Wounds UK 2006; 2: 92-101.

13. Edwards H, Courtney M, Finlayson K, Shuter P, Lindsay E. A randomised controlled trial of a community nursing intervention: improved quality of life and healing for clients with chronic leg ulcers. J Clin Nurs 2009; 18 : 1541-9.

14. Van Hecke A, Grypdonck M, Beele H, Vanderwee K, Defloor T. Adherence to leg ulcer lifestyle advice: qualitative and quantitative outcomes associated with a nurse-led intervention. J Clin Nurs 2011; 20: 429-43.

15. Moffatt CJ. Sociodemographic factors in chronic leg ulceration. BJD 2006; 155: 307-12.

16. Reich-Schupke S, Murmann F, Altmeyer P, Stucker M. Quality of life and patients' view of compression therapy. Int Angiol 2009; 28: 385-93.

17. Schwartzmann L. Calidad de Vida Relacionada con la Salud: Aspectos Conceptuales. Ciencia y Enfermería 2003; 2: 9-21.

18. Robinson Ramírez F. Calidad de vida relacionada con la salud como medida de resultados en salud: revisión sistemática de la literatura. Rev Colomb Cardiol 2007; 14: 207-22.

19. Cella DF. Measuring quality of life in palliative care. Sem Oncol 1995; 22: 73-8.

20. Palomino Medina M, Tárraga López P, Robayna E, López Cara M. Tratamiento de la insuficiencia venosa crónica y calidad de vida en una Zona Básica de Salud. Medicina General 2006; 86: 452-63.

21. Augustin M. Validation of a comprehensive Freiburg Life Quality Assessment (FLQA) core questionnaire. Eur J Dermatol 2004; 14: 107-13.

22. Urzúa A. Calidad de vida relacionada con la salud: Elementos conceptuales. Rev Med Chile 2010; 138: 358-65.
23. Ebeeskog B, Ekman S. Elderly persons experiences of living with venous leg ulcer. Scand J Caring Sci 2001; 15: 235-43.

24. Puffett N, Martin L, Kiew Chow M. Cohesive shortstretch vsfour-layer bandages for venous leg ulcers. Wound Care 2006.

25. U.S. Department of Health Services, Food and Administration, Center for Biologics Evaluation and Research, Guidance for Industry Patient Reported Outcome Measures: Use in Medical Product Development to Support Labeling Claims, 2009. Disponible en: http://www.fda. gov/Drugs/GuidanceComplianceRegulatoryInformation/Guidances/default.htm(CDER). [Consultado el 11 de noviembre de 2014].

26. Lamping DL, Schroter S, Kurz X, Kahn SR. Evaluation of outcomes in chronic venous disorders of the leg: Development of a scientifically rigorous, patient-reported measure of symptoms and quality of life. J Vasc Surg 2003; 37: 410-9.

27. Iglesias C, Birks Y, Nelson EA, Scanlon E. Health-Related Quality of Life in Patients with Venous Ulceration. Phlebology 2001; 16: 12-6.

28. Soon SL, Chen S. What are Wound Care Outcomes? Wounds 2004; 16: 2-6.

29. Iglesias CP, Birks Y, Nelson EA, Scanlon E, Cullum NA. Quality of life of people with venous leg ulcers: a comparison of the discriminative and responsive characteristics of two generic and a disease specific instruments. Qual Life Res 2005; 14 (7): 1705-18.

30. Smith JJ, Guest MG, Greenhalgh RM, Davies AH. Measuring thequality of life in patients with venous ulcers. J Vasc Surg 2000; 31: 642-9.

31. Chase SK, Whittemore R, Crosby N, Freney D. Living with Chronic Venus Legs ulcers: a descriptive study of knowledge and funtional health status. J Community Health Nurs 2000; 17 (1): 1-13.

32. Iglesias C, Nelson EA, Cullum NA, Torgerson DJ. A randomised controlled trial of two types of bandage for treating venous leg ulcers. Heal Tech Assess 2004; 8 (29): $1-105$.

33. Vilagut G, Ferrer M, Rajmil L, Rebollo P, Permanyer-Miralda G, Quintana JM. El Cuestionario de Salud SF-36 español: una década de experiencia y nuevos desarrollos. Gac Sanit 2005; 19 (2): 135-50.

34. Vásquez MA, Munschauer CE. Venous Clinical Severity Score and quality-of-life assessment tools: application to vein practice. Phlebology 2008; 23: 259-75.

35. Walters SJ, Morrell CJ, Dixon S. Measuring Health-related quality of life in patients with venous leg ulcers. Qual Life Res 1999; 8 (4): 327-36.

36. Howard A, Davies AH. Health-Related Quality of Life in 
Patients with Venous Ulceration. Phlebology 2001; 16.

37. Alonso J, Anto JM, Moreno CI. Spanish Version of the Nottingham Health Profile: Translation and Preliminary Validity. AJPH 1990; 80 (6): 704-8.

38. Palfreyman SJ, Tod AM, Brazier JE, Michaels JA. A systematic review of health-related quality of life instruments used for people with venous ulcers: an assessment of their suitability and psychometric properties. J Clin Nurs 2010; 19: 2673-703.

39. Reeve BB, Hays RD, Bjorner JB, Cook F, Crane PK, Teresi J. Psychometric Evaluation and Calibration of Health-Related Quality of Life Item Banks. Med Care 2007; 45: S22-S31.

40. Cella D, Yount S, Rothrock N, Gershon R, Cook K, Reeve B. The Patient-Reported Outcomes Measurement Information System (PROMIS). Med Care 2007; 45: S3S11.

41. The World Health Organization Quality of Life assessment (WHOQOL): position paper from the World Health Organization. Soc Sci Med 1995; 41 (10): 14039.

42. Espinoza I, Osorio P, Torrejón MJ, Lucas-Carrasco R, Bunout D. Validación del cuestionario de calidad de vida (WHOQOL-BREF) en adultos mayores chilenos. Rev Med Chile 2011; 139: 579-86.

43. González-Consuegra RV, Verdú J. Proceso de adaptación al castellano del Charing Cross Venous Ulcer Questionnaire (CCVUQ) para medir la calidad de vida relacionada con la salud en pacientes con úlceras venosas. Gerokomos 2010; 21 (2): 80-7.

44. González-Consuegra RV. Calidad de vida y cicatrización en pacientes con úlceras de etiología venosa. Validación del Charing Cross Venous Ulcer Questionnaire, versión española (CCVUQ-e) y del Pressure Ulcer Scale for Healing, versión española (PUSH-e). Gerokomos 2011; 22 (3): 131-6.

45. Hareendran A, Doll H, Wild DJ, Moffatt CJ, Musgrove E, Wheatley C, et al. The venous leg ulcer quality of life (VLU-QoL) questionnaire: development and psychometric validation. Wound Repair Regen 2007; 15 (4): 465-73.

46. Sánchez R, Echeverry J. Validación de Escalas de Medición en Salud. Rev Salud pública 2004; 6: 302-18.

47. Lizán L, Paz S, Dilla T, Rodríguez JM, Lahoz R, Reig-Ferrer A. Resultados en Salud Percibidos por el Paciente. Concepto e Instrumentos. Aten Primaria 2009; 41 (5): 275-80.

48. Jull A, Parag V, Walker N, Rodgers A. Responsiveness of generic and disease-specific health-related quality of life instruments to venous ulcer healing. Wound Rep Reg 2010; 18 (1): 26-30.
49. Fries JF, Bruce B, Cella D. The promise of PROMIS: Using item response theory to improve assessment of patient-reported outcomes. Clin Exp Rheumatol 2005; 23: S53-7.

50. Wong I, Lee D, Thompson D. Translation and validation of the Chinese version of the Charing Cross Venous Ulcer Questionnaire. J Clin Nurs 2006; 15: 356-7.

51. MAPI Research Trust. http://www.mapi-trust.org/ressources/questionnairestranslations. [Consultado el 11 de noviembre de 2014].

52. Haysm R, Bjorner J, Spritzer K, Cella D. Development of physical and mental health summary scores from Patient Reported Outcomes Measurement Information System (PROMIS) global items. Qual Life Res 2009; 18 (7): 873-80.

53. Deborah N. Developing the Patient-Reported Outcomes Measurement Information System (PROMIS). Med Care 2007; 45: S3-S11.

54. Wild D, Grove A, Martin M, Eremenco S, McElroy S, Verjee-Lorenz A, , et al. ISPOR Task Force for Translation and Cultural Adaptation. Principles of Good Practice for the Translation and Cultural Adaptation Process for Patient-Reported Outcomes (PRO) Measures: report of the ISPOR Task Force for Translation and Cultural Adaptation. Value Health 2005; 8 (2): 94-104.

55. Flaherty JA, Gaviria FM, Pathak D, Mitchell T, Wintrob R, Richman JA, et al. Developing instruments for cross-cultural psychiatry research. J Nerv Ment Dis 1988; 176 (5): 257-63.

56. Fernández G, Dallo MA, Durán C, Caperchione F, Gutiérrez S, Dapueto JJ. Cuestionario sobre Calidad de Vida Pediátrica (PedsQL) versión 4.0: fase inicial de la adaptación transcultural para Uruguay. Arch Pediat Urug 2010; 81 (2): 91-9.

57. Lent L, Hahn E, Eremenco S, Webster K, Cella D. Using cross-cultural input to adapt the Functional Assessment of Chronic Illness Therapy (FACIT) scales. Acta Oncol 1999; 38 (6): 695-702.

58. Herdman M, Fox-Rushby J, Badía X. Equivalence' and the translation and adaptation of health-related quality of life questionnaires. Qual Life Res 1997; 6: 37-47.

59. Fernández Montequín JI. Difficult healing wounds. Rev Cubana de Angiología y Cirugía Vascular 2012; 13 (1). Disponible en: http://bvs.sld.cu/revistas/ang/ vol13_1_12/angsu112.htm. [Consultado el 11 de noviembre de 2014].

60. Dapueto JJ, Servente L, Francolino C, Hahn EA. Determinants of Quality of Life in Patients with Cancer. Cancer 2005; 103: 1072-81.

61. Dapueto JJ, Fiori N, De Souza L, Dutra I, Ramírez R, Antiga C. Self-reported measures of general perception 
of health, psychological well-being, and risk factors at work helped to define promotional and preventive mental health interventions. Qual Life Res 2013; 22 (Suppl. $1): 47$.

62. Jason J. Smith JJ, Guest M, Greenhalgh R, MChir, FRCS. Measuring the quality of life in patients with venous ulcers. J Vasc Surg 2000; 31: 642-9.

63. Simon DA, Dix FP, McCollum CN. Management of venous leg ulcers. Br Med J 2004; 328 (7452): 1358-62.

64. Moffatt CJ, Franks PJ, Doherty DC, Martin R, Blewett $\mathrm{R}$, Ross F. Prevalence of leg ulceration in a London population. Q J Med 2004; 97: 431-7.

65. Vowden P, Apelqvist J, Moffatt C. Wound complexity and healing. Disponible en: www.ewma.org/english/ publications/documents/all-documents [Consultado el
11 de junio de 2013].

66. Grey JE, Harding KG, Enock S. Venous and arterial ulcers. BMJ 2006; 332: 347-50.

67. Araújo da Silva FA, Magalhães Moreira TA. Sociodemografic and clinical characteristics of customers with venous leg ulcer. Rev Enferm UERJ 2011; 19 (3): 468-72.

68. Callam MJ, Harper D, Rucklet C. Chronic leg ulceration: socio-economic aspects. Scott Med J 1988; 33: 358-60.

69. Moffatt CJ. Sociodemographic factors in chronic leg ulceration. BJD 2006; 155: 307-12.

70. Romanelli M, Vuerstaek JD, Rogers LC, Armstrong DG, Apelqvist J. Carga económica de las heridas de difícil cicatrización. Disponible en: www.ewma.org/english/ publications/documents/all-documents. [Consultado el 11 de junio de 2013]. 